\title{
Application of vector analysis on study of illuminated area and Doppler characteristics of airborne pulse radar
}

\author{
Haijiang Wang ${ }^{1,2^{*}}$ and Ling Yang ${ }^{1}$
}

\begin{abstract}
In this paper, the application of vector analysis tool in the illuminated area and the Doppler frequency distribution research for the airborne pulse radar is studied. An important feature of vector analysis is that it can closely combine the geometric ideas with algebraic calculations. Through coordinate transform, the relationship between the frame of radar antenna and the ground, under aircraft motion attitude, is derived. Under the time-space analysis, the overlap area between the footprint of radar beam and the pulse-illuminated zone is obtained. Furthermore, the Doppler frequency expression is successfully deduced. In addition, the Doppler frequency distribution is plotted finally. Using the time-space analysis results, some important parameters of a specified airborne radar system are obtained. Simultaneously, the results are applied to correct the phase error brought by attitude change in airborne synthetic aperture radar (SAR) imaging.
\end{abstract}

Keywords: Vector analysis; Illuminated area; Doppler characteristics

\section{Introduction}

In a radar system, it is necessary to describe the time-space relation as clearly as possible. The radar geometry study is very important for the deduction of radar echo signal. For example, in airborne radar, if it is required to present the radar echo equation and to obtain the illuminated area with Doppler characteristics, the geometry relations among the airplane, the antenna, and the ground must be analyzed intensively.

Researchers have done some studies on the illumination characteristics of radar. In [1], Korkmaz and Genderen measured the footprint of stepped frequency continuous wave $(\mathrm{CW})$ radar using a probe antenna, but little geometry and time-space analysis are applied. In [2], for airborne radar, Green et al. analyzed the attitude changes and their influences on the echo's Doppler spectrum but the timespace analysis was rarely applied and the illuminated area was not obtained. In [3], Liu and Arcone calculated the radar pulse's wave field on the ground with pseudospectral

\footnotetext{
* Correspondence: whi@cuit.edu.cn

${ }^{1}$ College of Electronic Engineering, Chengdu University of Information Technology, Chengdu, Sichuan 610225, China

${ }^{2}$ CMA. Key Laboratory of Atmospheric Sounding, Chengdu, Sichuan 610225,
} China

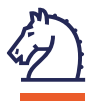

time domain method. This article is written on the hypothesis that the antenna's shape is known and its position is fixed, but there is no further discussion on the radar platform's motion and attitude changes.

The concept and calculation of a vector were detailed in textbooks since early times [4-6], and vector analysis has been used in space geometry, computer graphics, and field analysis [7-10]. But it has not been used sufficiently in the study of airborne radar geometry. Many researchers just refer to the vector method for show in the beginning and at the end of discussions, but during the process, they often rely on figure observation rather than vector calculation to derive equations [11-13]. In fact, making the full advantage of vector analysis in geometry attituderelated problems can make geometry concepts clearer and improve research efficiency noticeably. In addition, vectorial expressions can be transferred to MATLAB statements directly, which are convenient for the vector calculation and figure plotting.

In this paper, the vector analysis is adopted to study the geometry configuration of airborne pulse radar. Based on the vector derivation, some important results about the time-space relations of the airborne radar signal are 
obtained. These results will be applied to solve some problems in airborne radar application.

The paper is organized as follows. In Section 2, under the hypothesis of the airplane's attitude, the relationship between antenna coordinate frame and ground coordinate frame is deduced. Next, in Section 3, through the analysis of transmitted and received signal paths of the radar, according to the vector differential equation, a complete expression of the radar echo is obtained. Furthermore, the echo area of the airborne pulse radar and the echo's Doppler distribution on the ground are calculated in Section 4. In addition, the geometry characteristics of airborne radar signal transmission are applied to obtain parameters of airborne radar, and those features are adopted for the phase error correction in airborne synthetic aperture radar (SAR) imaging in Section 5 . Lastly, the conclusion is presented in Section 6.

\section{The relationship of the coordinate frames}

In this paper, it is assumed that ground frame is formed by unit vectors $\hat{X}, \hat{Y}$, and $\hat{Z}$. In the coordinate, $\hat{X}$ and $\hat{Y}$ are parallel to the ground level and $\hat{Z}$ is perpendicular to it. These three unit vectors form a right-handed system as follows:

$$
\hat{Z}=\hat{X} \times \hat{Y} \text {. }
$$

It is designed that the aircraft frame is formed by unit vectors $\hat{F}$ (along the fuselage direction), $\hat{W}$ (along the wing direction), and $\hat{T}$ (along the top direction), which form another right-handed helix system. So, they can be expressed as

$$
\hat{T}=\hat{F} \times \hat{W} .
$$

It is assumed the initial attitude of the aircraft is

$$
\left[\begin{array}{c}
\hat{F}^{\prime} \\
\hat{W}^{\prime} \\
\hat{T}^{\prime}
\end{array}\right]=\left[\begin{array}{lll}
1 & 0 & 0 \\
0 & 1 & 0 \\
0 & 0 & 1
\end{array}\right]\left[\begin{array}{c}
\hat{X} \\
\hat{Y} \\
\hat{Z}
\end{array}\right],
$$

and the angle of the aircraft which rotates along the top axis $\hat{T}$ is supposed to be $\psi$, which is known as the azimuth angle. Through the vector calculation, the new attitude can be obtained as follows:

$$
\left[\begin{array}{c}
\hat{F}^{\prime \prime} \\
\hat{W}^{\prime \prime} \\
\hat{T}^{\prime \prime}
\end{array}\right]=\left[\begin{array}{ccc}
\cos \psi & \sin \psi & 0 \\
-\sin \psi & \cos \psi & 0 \\
0 & 0 & 1
\end{array}\right]\left[\begin{array}{c}
\hat{F}^{\prime} \\
\hat{W}^{\prime} \\
\hat{T}^{\prime}
\end{array}\right]=P(\psi)\left[\begin{array}{c}
\hat{X} \\
\hat{Y} \\
\hat{Z}
\end{array}\right] .
$$

On the above basis, it is supposed that the aircraft rotates along the new wing axis $\hat{W}^{\prime \prime}$ by an angle $\alpha$, which is called the elevation angle, and the attitude of the aircraft transfers to

$$
\left[\begin{array}{c}
\hat{F}^{\prime \prime \prime} \\
\hat{W}^{\prime \prime \prime} \\
\hat{T}^{\prime \prime \prime}
\end{array}\right]=\left[\begin{array}{ccc}
\cos \alpha & 0 & \sin \alpha \\
0 & 1 & 0 \\
-\sin \alpha & 0 & \cos \alpha
\end{array}\right]\left[\begin{array}{c}
\hat{F}^{\prime \prime} \\
\hat{W}^{\prime \prime} \\
\hat{T}^{\prime \prime}
\end{array}\right]=A(\alpha) P(\psi)\left[\begin{array}{c}
\hat{X} \\
\hat{Y} \\
\hat{Z}
\end{array}\right] .
$$

On the basis of the above two rotations, suppose that the aircraft rotates once more along the fuselage axis by an angle $\beta$, which is called the rolling angle, and the attitude is obtained as

$$
\left[\begin{array}{c}
\hat{F} \\
\hat{W} \\
\hat{T}
\end{array}\right]=\left[\begin{array}{ccc}
1 & 0 & 0 \\
0 & \cos \beta & -\sin \beta \\
0 & \sin \beta & \cos \beta
\end{array}\right]\left[\begin{array}{c}
\hat{F}^{\prime \prime \prime} \\
\hat{W}^{\prime \prime \prime} \\
\hat{T}^{\prime \prime \prime}
\end{array}\right]=B(\beta) A(\alpha) P(\psi)\left[\begin{array}{c}
\hat{X} \\
\hat{Y} \\
\hat{Z}
\end{array}\right] .
$$

For the angles above, the range of elevation angle $\alpha$ is within $[0 \pi)$ and the ranges of azimuth angle $\psi$ and rolling angle $\beta$ are both within $[02 \pi)$. These three angles determine all possible attitudes of the aircraft.

The antenna frame can be derived from the aircraft frame as

$$
\begin{aligned}
& {\left[\begin{array}{l}
\hat{x} \\
\hat{y} \\
\hat{z}
\end{array}\right]=\left[\begin{array}{ccc}
1 & \cos \left(\gamma+\frac{\pi}{2}\right) & -\sin \left(\gamma+\frac{\pi}{2}\right) \\
0 & \cos \left(\gamma+\frac{\pi}{2}\right) \\
0 & \sin \left(\gamma+\frac{\pi}{2}\right) & \cos \left(\gamma+\frac{\pi}{2}\right)
\end{array}\right]\left[\begin{array}{c}
\hat{F} \\
\hat{W} \\
\hat{T}
\end{array}\right]} \\
& =\left[\begin{array}{ccc}
1 & 0 & 0 \\
0 & -\sin \gamma & -\cos \gamma \\
0 & \cos \gamma & -\sin \gamma
\end{array}\right]\left[\begin{array}{c}
\hat{F} \\
\hat{W} \\
\hat{T}
\end{array}\right]=Q(\gamma)\left[\begin{array}{c}
\hat{F} \\
\hat{W} \\
\hat{T}
\end{array}\right] \text {, }
\end{aligned}
$$

where $\gamma$ is the depression angle of the antenna's main lobe, $\hat{z}$ represents the direction of the main lobe's center line, $\hat{x}=\hat{F}$ is along the fuselage direction, and $\hat{y}=\hat{z} \times \hat{x}$. So, the antenna frame is obtained by rotating the aircraft frame at the angle of $\gamma+\frac{\pi}{2}$ along the fuselage direction.

From the above discussion, the relationship between antenna frame and ground frame can be expressed as

$$
\begin{aligned}
{\left[\begin{array}{l}
\hat{x} \\
\hat{y} \\
\hat{z}
\end{array}\right] } & =Q(\gamma) B(\beta) A(\alpha) P(\psi)\left[\begin{array}{c}
\hat{X} \\
\hat{Y} \\
\hat{Z}
\end{array}\right] \\
& =\Pi(\alpha, \beta, \gamma, \psi)\left[\begin{array}{c}
\hat{X} \\
\hat{Y} \\
\hat{Z}
\end{array}\right] .
\end{aligned}
$$

It is deduced as follows:

$$
\begin{aligned}
Q(0) & =B(0)=A(0)=P(0)=\overleftrightarrow{I} \\
& =\left[\begin{array}{lll}
1 & 0 & 0 \\
0 & 1 & 0 \\
0 & 0 & 1
\end{array}\right] .
\end{aligned}
$$

Through the algebra calculation, it can be obtained as follows: 


$$
\begin{aligned}
Q(\gamma) B(\beta) & =\left[\begin{array}{ccc}
1 & 0 & 0 \\
0 & \sin (\beta-\gamma) & -\cos (\beta-\gamma) \\
0 & \cos (\beta-\gamma) & \sin (\beta-\gamma)
\end{array}\right] \\
& =C(\beta-\gamma) .
\end{aligned}
$$

In order to keep the generality, the azimuth angle can be assumed as $\psi=0$. Then, the final result can be obtained as

$$
\begin{aligned}
\Pi(\alpha, \beta, \gamma, 0) & =Q(\gamma) B(\beta) A(\alpha)=C(\beta-\gamma) A(\alpha) \\
& =\left[\begin{array}{ccc}
\cos \alpha & 0 & \sin \alpha \\
\cos (\beta-\gamma) \sin \alpha & \sin (\beta-\gamma) & -\cos (\beta-\gamma) \cos \alpha \\
-\sin (\beta-\gamma) \sin \alpha & \cos (\beta-\gamma) & \sin (\beta-\gamma) \cos \alpha
\end{array}\right]
\end{aligned}
$$

\section{Expression of the airborne pulse radar echo signals}

Before considering the expression of the airborne pulse radar echo signal, the formula for the differential of a vector's magnitude is necessary to deduce.

For any vector $a$,

$$
|a|^{2}=a \cdot a \text {. }
$$

Conducting the differential operation on both sides of the above equation, it is transferred to

$$
2|a| d|a|=2 a \cdot d a .
$$

So,

$$
d|a|=\frac{a}{|a|} \cdot d a=\hat{a} \cdot d a .
$$

This result is very important and will be used later.

The transmission and reception of a ray during the flight of the aircraft are shown in Figure 1. Assume that the position vector of the aircraft is as follows:

$$
R(t)=R_{0}+t v,
$$

where $v$ is the velocity vector.

Assume that a ray in the radar beam is transmitted at the zero time and reaches the ground point and the length of the ray is

$$
l_{1}=\left|r_{0}\right|=\left|r_{s}-R(0)\right| .
$$

If the reflected signal arrived to the radar at time $t$, then the distance that the reflected signal traverses is

$$
l_{2}=|r(t)|=\left|r_{s}-R(t)\right| .
$$

Suppose the transmitted pulse signal of the airborne radar is

$$
S(t)=A_{m}(t) e^{j \omega t},
$$

where

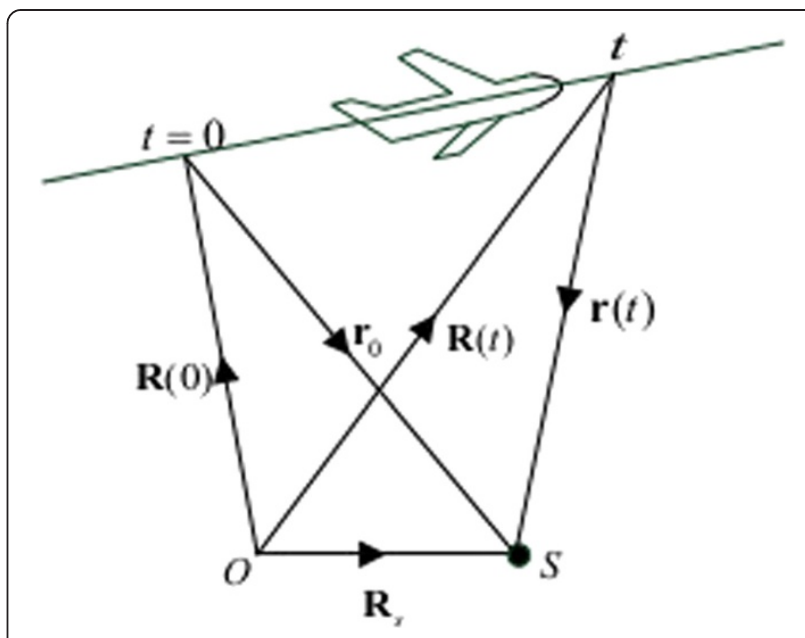

Figure 1 Process of transmission and reception of a ray.

$$
A_{m}(t)=\left\{\begin{array}{cc}
1 & 0 \leq t \leq \tau \\
0 & \text { elsewhere }
\end{array}\right.
$$

The time delay between the received signal and the transmitted signal is

$$
t_{d}(t)=\frac{l_{1}+l_{2}}{c}=\frac{1}{c}\left[\left|r_{s}-R(0)\right|+\left|r_{s}-R(t)\right|\right] .
$$

For the radar's motion, $l_{1}, l_{2}$, and $t_{d}$ are related to $t$. According to Equation 11, the differential coefficient of $t_{d}$ is

$$
\frac{d t_{d}}{d t}=-\frac{1}{c}\left[\frac{\left[r_{s}-R(0)\right]}{\left|r_{s}-R(0)\right|}+\frac{\left[r_{s}-R(t)\right]}{\left|r_{s}-R(t)\right|}\right] \cdot v .
$$

It is obvious that the time delay at the zero time is obtained by

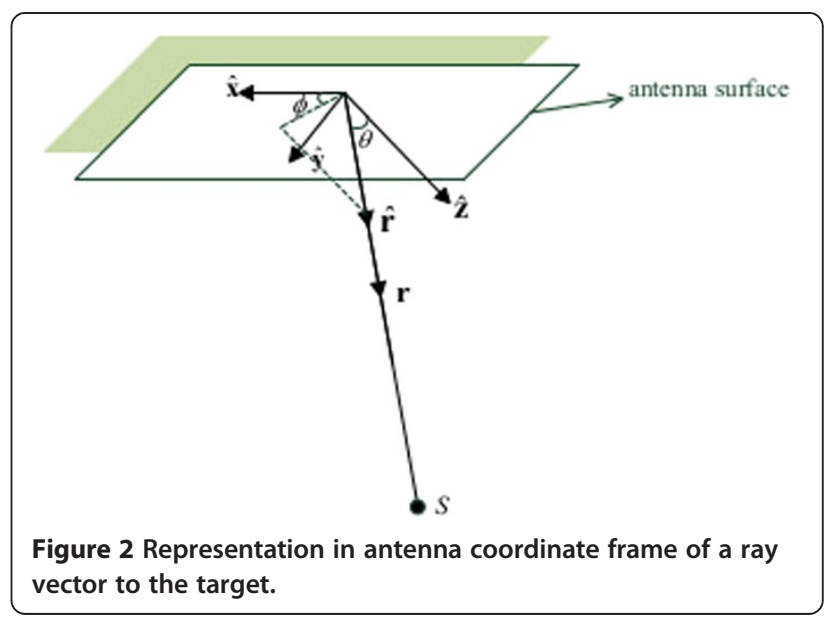


Wang and Yang EURASIP Journal on Advances in Signal Processing 2014, 2014:114

Page 4 of 9

http://asp.eurasipjournals.com/content/2014/1/114

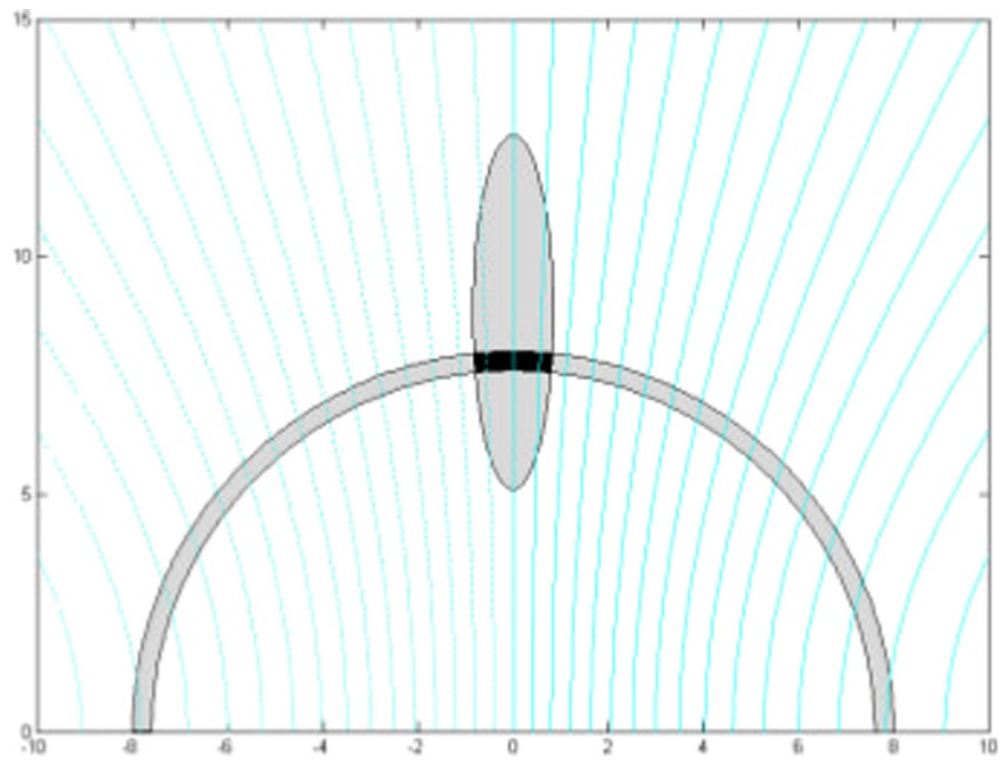

Figure 3 Illuminated area and Doppler frequency distribution when $\alpha=0, \beta=0, \gamma=45^{\circ}, \sigma_{x}=5^{\circ}, \sigma_{y}=15^{\circ}$, and $H=5 \mathrm{~km}$.

$$
t_{d}(0)=\frac{2 r}{c} ;\left.\quad \frac{d t_{d}}{d t}\right|_{t=0}=-\frac{2}{c} \hat{r} \cdot v=-\frac{2 v_{r}}{c} .
$$

So, the one-order expansion of $t_{d}$ can be obtained as

$$
t_{d}(t) \approx \frac{2 r}{c}-\frac{2 v_{r}}{c} t
$$

The second item in the above equation is much smaller than the first item, so in the amplitude expression, it can be neglected as

$$
A_{m}\left(t-t_{d}\right)=A_{m}\left(t-\frac{2 r}{c}\right) \text {. }
$$

But in the phase expression, the second item in Equation 23 cannot be neglected, because after being multiplied by $\omega$, it can also reach the magnitude order $\pi$. So, the following expression of the airborne pulse radar echo signal can be obtained as

$$
s(t)=\iint_{\Sigma} \frac{A_{m}\left(t-\frac{2 r}{c}\right) G^{2}(\phi, \theta) \Lambda\left(r_{s}\right) \exp \left[j \omega\left(\left(1+\frac{2 v_{r}}{c}\right) t-\frac{2 r}{c}\right)\right]}{r^{2}} d \Sigma .
$$

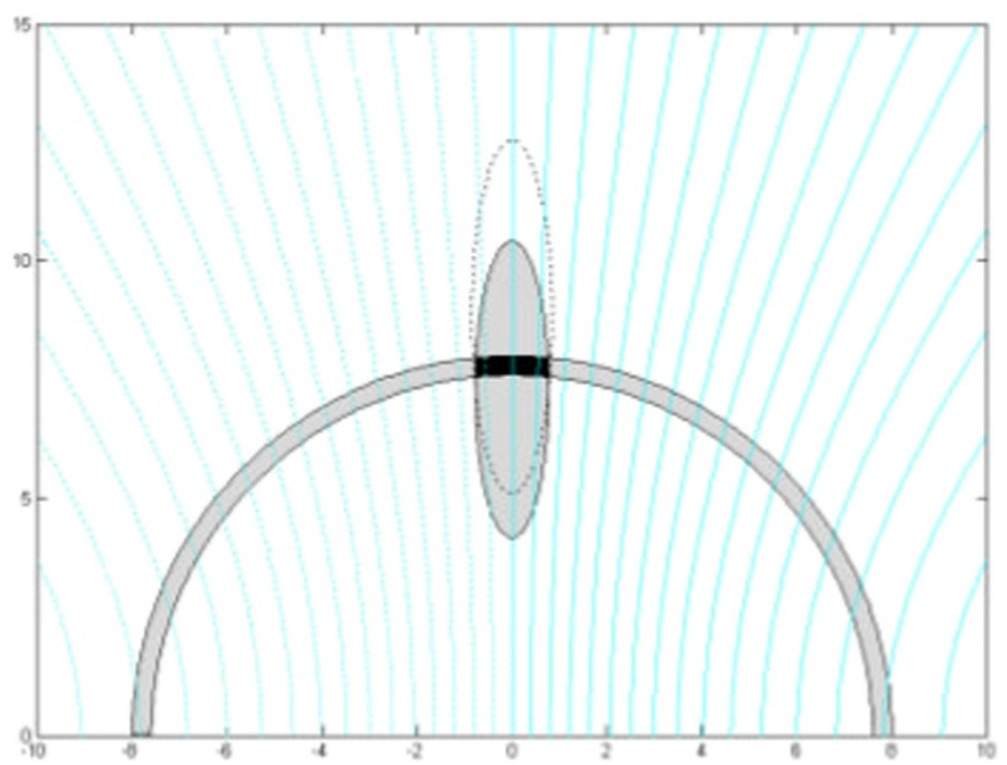

Figure 4 Illuminated area and Doppler frequency distribution when $\alpha=0, \beta=5^{\circ}, \gamma=45^{\circ}, \sigma_{x}=5^{\circ}, \sigma_{y}=15^{\circ}$, and $H=5 \mathrm{~km}$. 


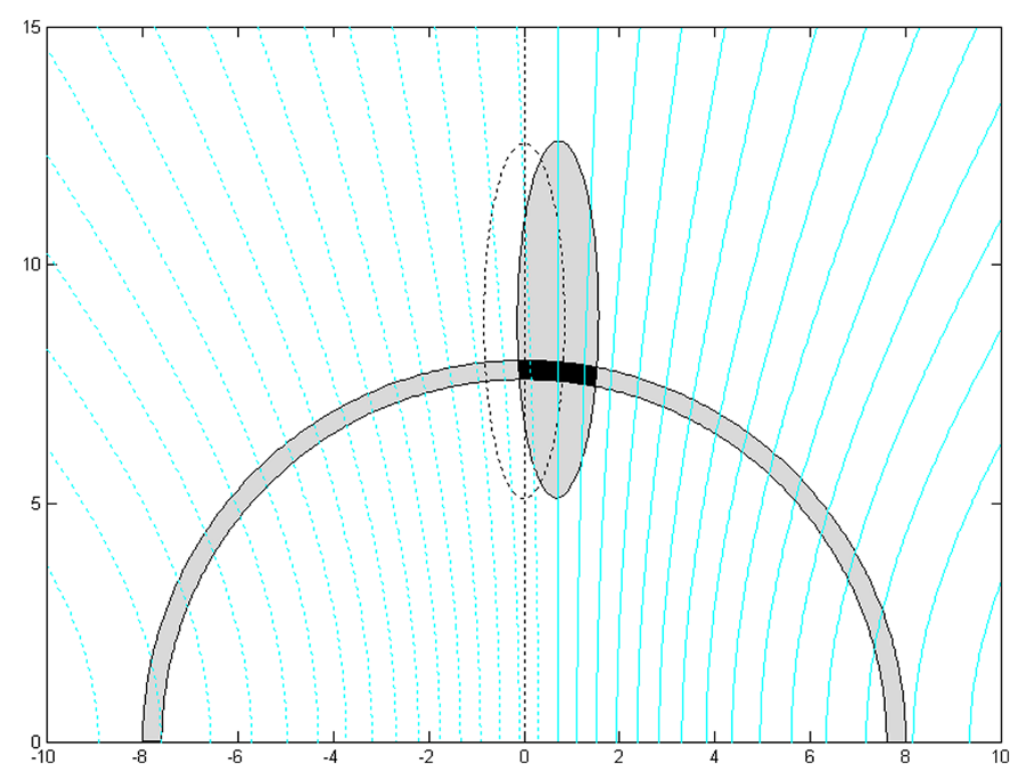

Figure 5 Illuminated area and Doppler frequency distribution when $a=5^{\circ}, \beta=0, \gamma=45^{\circ}, \sigma_{x}=5^{\circ}, \sigma_{y}=15^{\circ}$, and $H=5 \mathrm{~km}$.

4 The airborne pulse radar-illuminated area and the Doppler characteristic distribution

Expressing with the radar's antenna frame, the vector $\hat{r}$ is

$$
\hat{r}=\sin \theta \cos \phi \hat{x}+\sin \theta \sin \phi \hat{y}+\cos \theta \hat{z},
$$

which is shown in Figure 2 as

Because of $r_{s}=R(0)+r$ and $r_{s} \cdot \hat{Z}=0$, so it can be deduced as $[R(0)+r] \cdot \hat{Z}=\mathbf{0}$. Suppose $R(0)=H \hat{Z}$, then $r \cdot \hat{Z}=r \hat{r} \cdot \hat{Z}=-R(0) \cdot \hat{Z}=-H$. Based on Equation 26, $r$ can be obtained as

$$
\begin{aligned}
r & =-\frac{H}{\hat{r} \cdot \hat{Z}} \\
& =-\frac{H}{\sin \theta \cos \phi \sin \alpha-\sin \theta \sin \phi \cos \alpha \cos (\beta-\gamma)+\cos \theta \cos \alpha \sin (\beta-\gamma)} .
\end{aligned}
$$

Simultaneously, it is easy to determine the coordinate position of the ground reflection point as

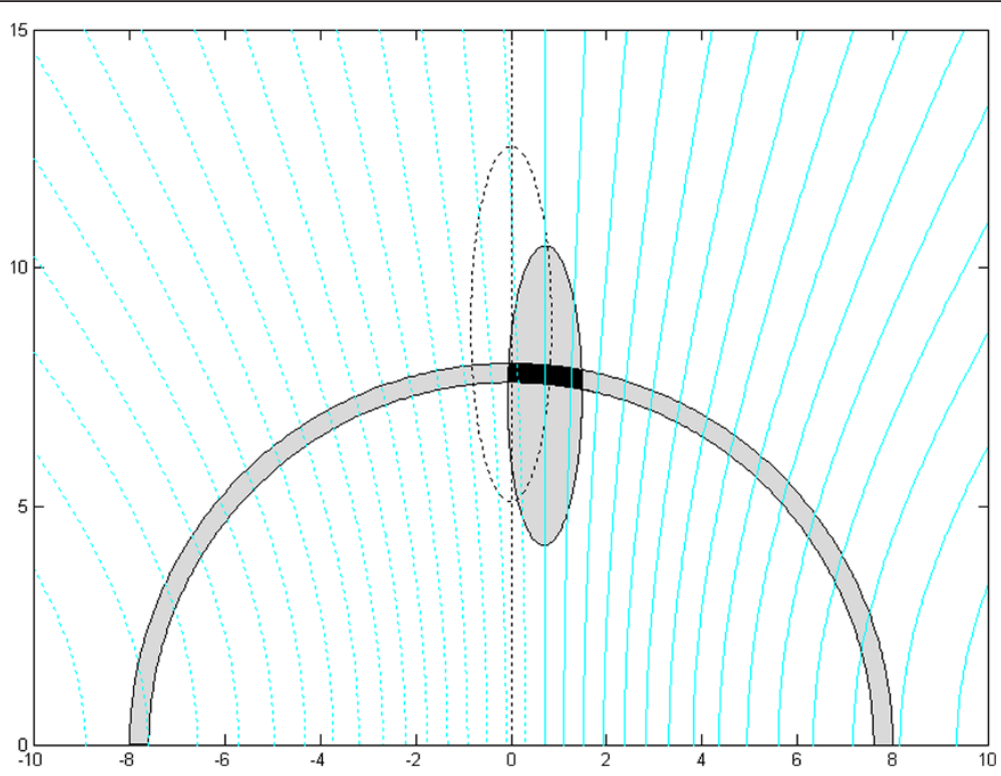

Figure 6 Illuminated area and Doppler frequency distribution when $a=5^{\circ}, \beta=5^{\circ}, \gamma=45^{\circ}, \sigma_{x}=5^{\circ}, \sigma_{y}=15^{\circ}$, and $H=5 \mathrm{~km}$. 


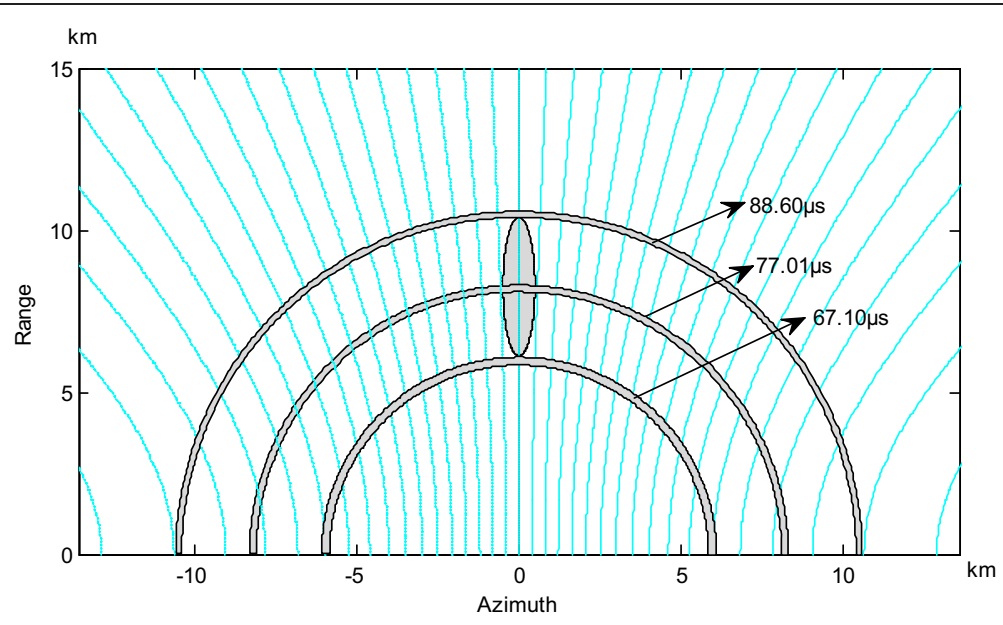

Figure 7 Illuminated area and Doppler distribution of an airborne pulse radar with known parameters.

$$
\begin{aligned}
X & =r_{s} \cdot \hat{X}=r \cdot \hat{X}=r \hat{r} \cdot \hat{X} \\
& =\frac{-H[\sin \theta \cos \phi \cos \alpha+\sin \theta \sin \phi \sin \alpha \cos (\beta-\gamma)-\cos \theta \sin \alpha \sin (\beta-\gamma)]}{\sin \theta \cos \phi \sin \alpha-\sin \theta \sin \phi \cos \alpha \cos (\beta-\gamma)+\cos \theta \cos \alpha \sin (\beta-\gamma)}, \\
Y & =r_{s} \cdot \hat{Y}=r \cdot \hat{Y}=r \hat{r} \cdot \hat{Y} \\
& =\frac{-H[\sin \theta \sin \phi \sin (\beta-\gamma)+\cos \theta \cos (\beta-\gamma)]}{\sin \theta \cos \phi \sin \alpha-\sin \theta \sin \phi \cos \alpha \cos (\beta-\gamma)+\cos \theta \cos \alpha \sin (\beta-\gamma)} .
\end{aligned}
$$

For the antenna's beam, the Gaussian model can be used as

$$
G(\phi, \theta)=G_{0} \exp \left[-\frac{\theta^{2}}{2}\left(\frac{\cos ^{2} \phi}{\sigma_{x}^{2}}+\frac{\sin ^{2} \phi}{\sigma_{y}^{2}}\right)\right] .
$$

Define the half-power area as

$$
G(\phi, \theta)=\frac{G_{0}}{\sqrt{2}} .
$$

From Equation 29, it can be obtained as

$$
\exp \left[-\frac{\theta^{2}}{2}\left(\frac{\cos ^{2} \phi}{\sigma_{x}^{2}}+\frac{\sin ^{2} \phi}{\sigma_{y}^{2}}\right)\right]=\frac{1}{\sqrt{2}}
$$

So,

$$
\frac{\theta^{2}}{2}\left(\frac{\cos ^{2} \phi}{\sigma_{x}^{2}}+\frac{\sin ^{2} \phi}{\sigma_{y}^{2}}\right)=\frac{\log 2}{2} .
$$

Express $\theta$ by $\phi$ as

$$
\theta=\sqrt{\frac{\sigma_{x}^{2} \sigma_{y}^{2} \log 2}{\sigma_{y}^{2} \cos ^{2} \phi+\sigma_{x}^{2} \sin ^{2} \phi}}
$$

The above equation indicates the relationship between $\theta$ and $\phi$, and the beam edge can be obtained from the equation.

Assume that $\phi$ varies within $[0,2 \pi)$ and substitute Equation 33 into Equation 28, and the beam outline on the ground can be obtained. The area inside the outline can be expressed as

$$
G(\phi, \theta) \geq \frac{G_{0}}{\sqrt{2}} .
$$

According to Equation 19, $A_{m}\left(t-\frac{2 r}{c}\right)$ is not zero only under the following condition:

$$
0 \leq t-\frac{2 r}{c} \leq \tau \text {. }
$$

So,

Table 1 Calculated parameters of the airborne pulse radar specified by Table 2

\begin{tabular}{llllll}
\hline Calculated parameters & Nearest range & Farthest range & Earliest reception time & Latest reception time & Doppler range \\
\hline Values & $6.1402 \mathrm{~km}$ & $10.4232 \mathrm{~km}$ & $67.10 \mu \mathrm{s}$ & $88.60 \mu \mathrm{s}$ & $\frac{2 v}{\lambda} 0.0986$ \\
\hline
\end{tabular}


Table 2 System parameters of an airborne pulse radar

\begin{tabular}{llllll}
\hline $\begin{array}{l}\text { System } \\
\text { parameters }\end{array}$ & $\begin{array}{l}\text { Flight } \\
\text { height }\end{array}$ & $\begin{array}{l}\text { Radar depression } \\
\text { angle }\end{array}$ & $\begin{array}{l}\text { Beam width in azimuth } \\
\text { direction }\end{array}$ & $\begin{array}{l}\text { Beam width in range } \\
\text { direction }\end{array}$ & $\begin{array}{l}\text { Pulse } \\
\text { duration }\end{array}$ \\
\hline Values & $5 \mathrm{~km}$ & $45^{\circ}$ & $3^{\circ}$ & $9^{\circ}$ & $1 \mu \mathrm{s}$ \\
\hline
\end{tabular}

$$
\frac{c(t-\tau)}{2} \leq r \leq \frac{c t}{2}
$$

Let $\rho=\sqrt{X^{2}+Y^{2}}=\sqrt{r^{2}-H^{2}}$, so the above equation can be rewritten as:

$$
\sqrt{\left[\frac{c(t-\tau)}{2}\right]^{2}-H^{2}} \leq \rho \leq \sqrt{\left(\frac{c t}{2}\right)^{2}-H^{2}}
$$

Equation 34 describes an area like a long ellipse, and Equation (37) describes a ring area. These two areas overlap to an area, which are shown in Figures 3, 4, 5, 6 and denoted by black color.

There is a Doppler frequency in the echo signal in Equation 25, which is expressed as

$$
f_{d}=\frac{2 \omega v_{r}}{2 \pi c}=\frac{4 \pi f v_{r}}{2 \pi c}=\frac{2 v_{r}}{\lambda}=\frac{2 v}{\lambda}(\hat{r} \cdot \hat{x}) .
$$

In Figures 3, 4, 5 and 6, $\hat{r} \cdot \hat{x}$, named as Doppler distribution, is plotted.

\section{Application of the above method and results}

In airborne pulse radar, when the system parameters, such as the platform height, radar beam width, and pulse duration, are determined, some other parameters can be calculated according to the above analysis method. Assume that the parameters are as follows:
By the above vector analysis method, the illuminated area and Doppler distribution are plotted, as shown in Figure 7.

Combining the vector calculation tool and figures, some important deductions, such as the reception parameters, can be obtained. According to Equations 28 and 33, the outline of the beam footprint can be obtained, so its nearest and farthest points can be acquired. On this basis, the reception time interval can be obtained accurately. Furthermore, according to Equation 38, the Doppler frequency of each point on the outline can be calculated, so the Doppler range of the beam footprint area can be obtained. In Table 1, some calculated parameters are listed for the radar platform, which is specified by the data in Table 2.

The above discussion indicates that by vector analysis, the geometry relationship of the airborne pulse radar is clear and some important parameters can be calculated.

In airborne SAR imaging, an invariable velocity and an unchanging attitude for the aircraft are expected for ensuring the image quality. But in the real experiment environments, inevitably, there are some attitude disturbances for various factors, such as air turbulence and instable steering. These attitude disturbances will change the illuminated area and its Doppler frequency distribution. And correspondingly, the echo signal will lose phase coherency with the reference signal and the

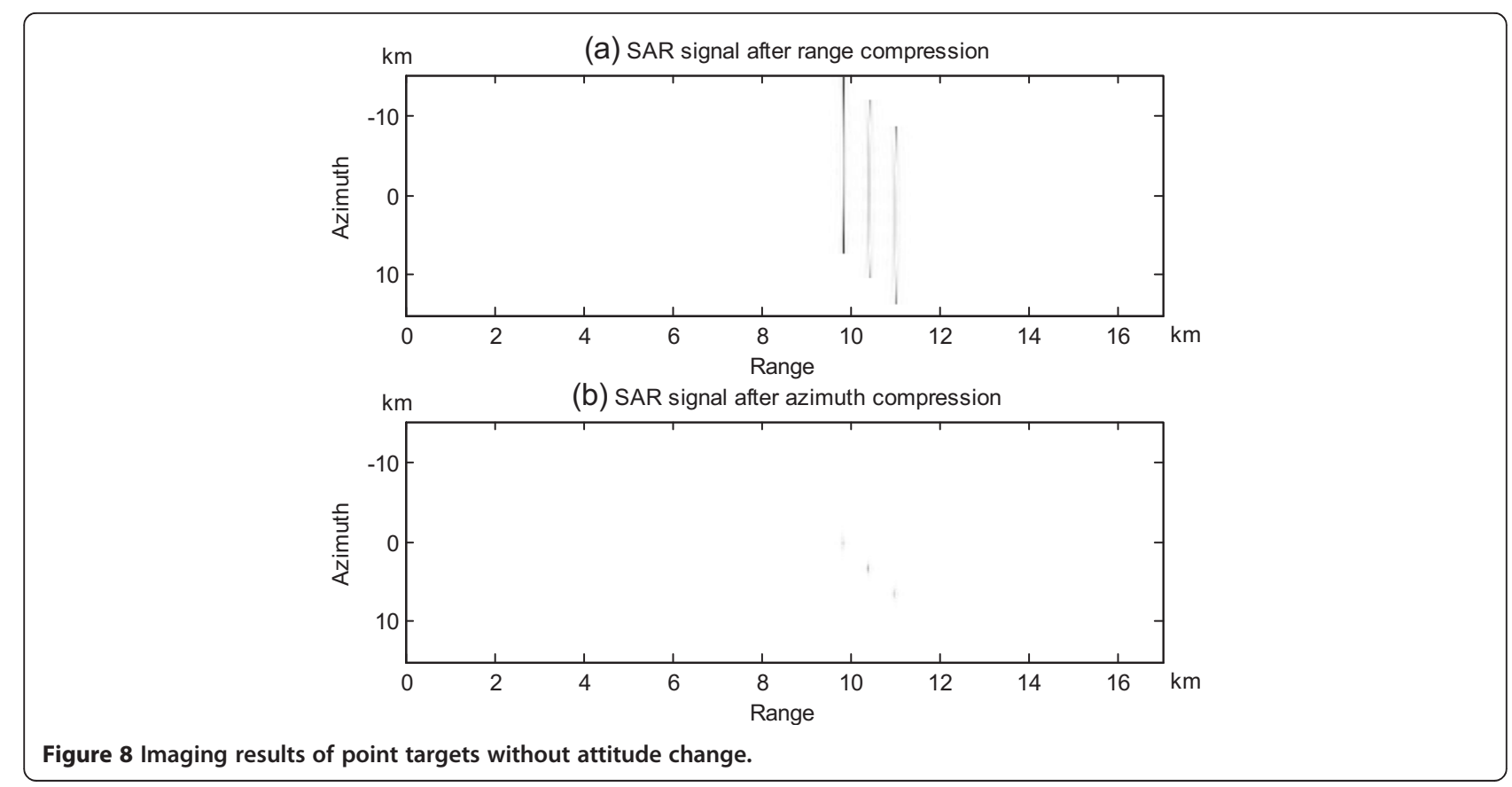



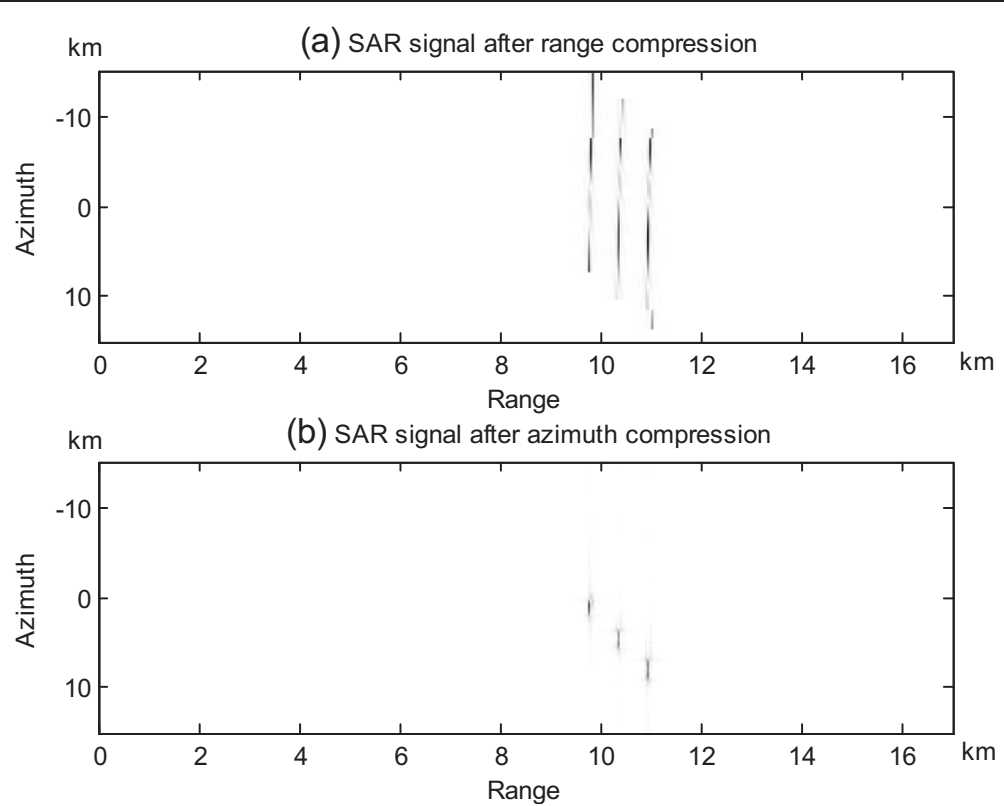

Figure 9 Imaging results of point targets with attitude change.

focusing performance of the SAR will be degraded. To solve this problem, several motion compensation methods and algorithms were proposed [14-17]. Most of these methods focus on the echo signal itself and rely on the estimation accuracy of Doppler centroid. In fact, the above research on illuminated area and Doppler frequency distribution can be applied to carry on phase error correction between the echo signals and the reference signals caused by radar platform motion.
In order to illustrate the influence of attitude change to SAR imaging, the imaging simulations are carried out for three point targets. Firstly, the case of no attitude change is conducted, and the imaging results are shown in Figure 8. Then, assume that the aircraft has an attitude change relative to the intended flight path and the pitching angle and the rolling angle are both set at $0.1^{\circ}$. Besides, a zero-mean white Gaussian noise with a variance of 0.005 is added to the angles. The simulated imaging results are shown in Figure 9.
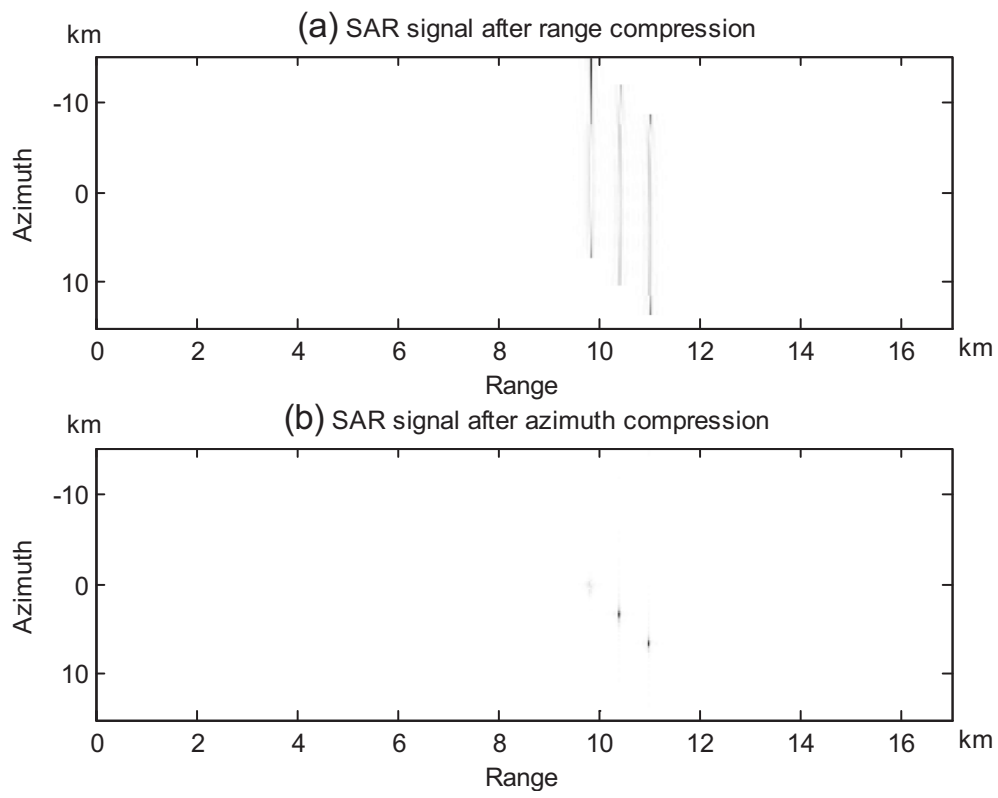

Figure 10 Imaging results of point targets after motion correction. 
From Figure 9, it is shown that there is a defocusing record in the imaging process when the radar platform's attitude keeps changing.

The real-time attitude is assumed to be recorded, but there is a zero-mean white Gaussian noise with a variance of 0.005 compared to the real value of the parameters, such as the pitching and rolling angle. In order to correct the imaging deviation, firstly, with the recorded attitude parameters, Doppler distribution is calculated by the vector analysis method proposed in Section 4. And then, according to the Doppler frequency and the distance to the radar antenna of each ground point, the phase of the reference signal is corrected. The imaging results with the corrected reference signal are shown in Figure 10.

Comparing Figure 10 to Figure 9, it is noticeable that the focusing performance is improved significantly after phase correction based on vector analysis.

\section{Conclusions}

In airborne pulse radar, some problems, especially the tasks involving geometry relationships, are difficult to solve by the scalar tool. However, as discussed in the proposed approach, the parameter results can be derived expediently and naturally by the vector method. From the discussion in the above sections, it can be seen that with the vector method, the time-space relationships, such as the expression of the echo signals, the illuminated area, and the Doppler characteristic distribution are obtained intuitively. Furthermore, the analysis results with the vector method can be applied in radar parameter calculation and SAR imaging. So, vector analysis is effective for airborne pulse radar parameter setting and solving and airborne pulse radar signal processing tasks.

Received: 28 February 2014 Accepted: 11 June 2014

Published: 19 July 2014

\section{References}

1. E Korkmaz, P Van Genderen, Antenna Footprint Measurements of Stepped Frequency CW Radar on the Air/Ground Interface, IEE Antenna Measurements and SAR (AMS'2004), 2004, pp. 87-91

2. JW Green, TB Hale, MA Temple, JT Buckreis, Incorporating Pulse-to-Pulse Motion Effects into Side-Looking Array Radar Models, Fourth IEEE Workshop on Sensor Array and Multichannel Processing, 2006, pp. 580-585

3. L Liu, SA Arcone, Near-Surface Radar Pulse Propagation in Complex Terrain Environments: Preliminary Results, 10th International Conference on Ground Penetrating Radar (2004), pp. 21-24

4. JG Coffin, Vector Analysis: an Introduction to Vector-Methods and Their Various Applications to Physics and Mathematics (J. Wiley \& sons, New York, 1911)

5. B Hague, An Introduction to Vector Analysis (Methuen \& Co. Ltd, London, 1951)

6. N Kemmer, Vector Analysis: A physicist's Guide to the Mathematics of Fields in Three Dimensions (Cambridge University Press, London, 1977)

7. $A B$ Sproul, Derivation of the solar geometric relationships using vector analysis. Renew. Energy 32(7), 1187-1205 (2007)

8. JR Miller, Vector geometry for computer graphics. IEEE Comput. Graph. Appl. 19(3), 66-73 (1999)

9. JR Miller, Applications of vector geometry for robustness and speed. IEEE Comput. Graph. Appl. 19(4), 68-73 (1999)

10. BM Notaros, Geometrical approach to vector analysis in electromagnetics education. IEEE Trans. Educ. 56(3), 336-345 (2013)
11. A Aprile, A Mauri, D Pastina, Real Time Rotational Motion Compensation Algorithm for Focusing Spot-SAR/ISAR Images in Case of Variable RotationRate, European Radar Conference(EURAD). 141-144 (2004)

12. J Balke, SAR Image Formation for Forward-Looking Radar Receivers in Bistatic Geometry by Airborne Illumination, IEEE Radar Conference, 2008, pp. 1-5

13. AD Lazarov, TP Kostadinov, SAR Signal Modeling and Imaging of a Moving Target, 2011 IEEE CIE International Conference on Radar, Vol. 1, 2001, pp. 510-513

14. P Guccione, C Cafforio, Motion Compensation Processing of Airborne SAR Data, IGARSS'2008 Vol. 3, 2008, pp. 1154-1157

15. Z Ding, L Liu, T Zeng, W Yang, T Long, Improved motion compensation approach for squint airborne SAR. IEEE Trans. Geosci. Remote. Sens. 51(8), 4378-4387 (2013)

16. YJ Zhang, KY Han, YP Wang, WX Tan, W Hong, Study on Motion Compensation for Airborne Forward Looking Array SAR by Time Division Multiplexing Receiving, Synthetic Asia-Pacific Conference on Aperture Radar (APSAR)2013, pp. 392-395

17. M Arii, Efficient motion compensation of a moving object on SAR imagery based on velocity correlation function. IEEE Trans. Geosci. Remote Sens. 52(2), 936-946 (2014)

doi:10.1186/1687-6180-2014-114

Cite this article as: Wang and Yang: Application of vector analysis on study of illuminated area and Doppler characteristics of airborne pulse radar. EURASIP Journal on Advances in Signal Processing 2014 2014:114.

\section{Submit your manuscript to a SpringerOpen ${ }^{\circ}$ journal and benefit from:}

- Convenient online submission

- Rigorous peer review

- Immediate publication on acceptance

- Open access: articles freely available online

- High visibility within the field

- Retaining the copyright to your article

Submit your next manuscript at $>$ springeropen.com 